\title{
An Optimization Algorithm for Separating Land Surface Temperature and Emissivity from Multispectral Thermal Infrared Imagery
}

\author{
Shunlin Liang, Member, IEEE
}

\begin{abstract}
Land surface temperature (LST) and emissivity are important components of land surface modeling and applications. The only practical means of obtaining LST at spatial and temporal resolutions appropriate for most modeling applications is through remote sensing. While the popular split-window method has been widely used to estimate LST, it requires known emissivity values. Multispectral thermal infrared imagery provides us with an excellent opportunity to estimate both LST and emissivity simultaneously, but the difficulty is that a single multispectral thermal measurement with $N$ bands presents $N$ equations in $N+1$ unknowns ( $N$ spectral emissivities and LST). In this study, we developed a general algorithm that can separate land surface emissivity and LST from any multispectral thermal imagery, such as moderate-resolution imaging spectroradiometer (MODIS) and advanced spaceborne thermal emission and reflection radiometer (ASTER). The central idea was to establish empirical constraints, and regularization methods were used to estimate both emissivity and LST through an optimization algorithm. It allows us to incorporate any prior knowledge in a formal way. The numerical experiments showed that this algorithm is very effective (more than $43.4 \%$ inversion results differed from the actual LST within $0.5^{\circ}, 70.2 \%$ within $1^{\circ}$ and $84 \%$ within $1.5^{\circ}$ ), although improvements are still needed.
\end{abstract}

Index Terms-Emissivity, land surface temperature, remote sensing, thermal infrared.

\section{INTRODUCTION}

$\mathbf{L}$ AND surface temperature (LST) is required for a variety of climatic, hydrologic, ecological, and biogeochemical studies [1]. Emissivities are strongly indicative of composition, especially for the silicate minerals that make up much of the land surface. They are also important for bedrock mapping and resource exploration [2]. Both are needed in the accurate calculation of outgoing longwave radiation emitted from the Earth's surface [3]. There have been many algorithms proposed and implemented for the retrieval of LST from remote observations. In particular, much research has focused on methods that use two thermal channels of the advanced very high resolution radiometer (AVHRR) sensor [4]-[6]. One common algorithm is the so-called split-window method, which has been used successfully for sea surface temperature retrievals [7], [8]. However, temperature derivation over land is more difficult than over the ocean because of the high spatial and temporal

Manuscript received December 6, 1999; revised March 29, 2000.

The author is with the Laboratory for Global Remote Sensing Studies, Department of Geography, University of Maryland, College Park, MD 20742 USA (e-mail: sliang@geog.umd.edu).

Publisher Item Identifier S 0196-2892(01)01787-9. variability of surface emissivity and atmospheric water vapor, both of which significantly affect the thermal radiance reaching the sensor (hence the recovered temperatures).

The central part of most split-window algorithms is based on the assumption that LST is linearly related to the brightness temperatures of two thermal channels. With the assumption that surface emissivities of these two channels are known, the split-window method can eliminate atmospheric effects for LST estimation. More studies have revealed that when the atmosphere is not particularly dry, the traditional split-window algorithm cannot remove the atmospheric effects completely. Many efforts (e.g., [9], [10]) therefore have been made to incorporate the column water vapor content of the atmosphere into the split-window formulae.

Estimating land surface emissivity at the global scale for being used in the split-window algorithms is very challenging. Several methods have been reported in the literature. One approach is to link emissivity with normalized difference vegetation index (NDVI) [11], [12]. Another approach is to associate it with land cover information [13] in which each cover type is assigned one emissivity value. Obviously, these two approaches cannot completely capture the tremendous amount of variability of surface emissivity, particularly over nonvegetated regions. An inaccuracy of only 0.01 in emissivity causes errors in LST exceeding those due to atmospheric correction [5]: "In fact the error due to an error in the emissivity correction is two times larger than that due to an error in the atmospheric correction ... Certainly this is an area of research that requires much more study"[14].

A very practical way for accurately estimating spectral emissivity is from thermal infrared imagery itself, which certainly requires multiple thermal channels. Fortunately, several spaceborne sensors will have multiple thermal infrared bands that enable us to estimate LST and emissivity simultaneously, such as MODIS and ASTER. The MODIS [15] has multiple thermal bands in the $3.5-4.2 \mu \mathrm{m}$ and the $8-13.5 \mu \mathrm{m}$ ranges, and the ASTER [16] has five thermal bands in the $8-12 \mu \mathrm{m}$ range (see Table I).

The split-window algorithms combine atmospheric correction and the LST estimation into one process. However, it is necessary to correct the atmospheric effects before separating temperature and emissivity from multiple thermal infrared imagery. The ASTER science team has proposed to correct the atmospheric effects using the atmospheric profiles produced by the MODIS sounders [17]. The MODIS science team proposed a day/night algorithm [1] that adjusts the MODIS atmospheric 
TABLE I

\begin{tabular}{l|c|c|c|c|c|c}
\hline Sensor & \multicolumn{6}{|c}{ Band $(\boldsymbol{\mu m})$} \\
\hline \multirow{2}{*}{ MODIS } & 20 & 22 & 23 & 29 & 31 & 32 \\
& $3.66-3.84$ & $3.929-3.989$ & $4.02-4.08$ & $8.4-8.7$ & $10.78-11.28$ & $11.77-12.27$ \\
\hline \multirow{2}{*}{ ASTER } & 10 & 11 & 12 & 13 & 14 & - \\
& $8.125-8.475$ & $8.475-8.825$ & $8.925-9.275$ & $10.25-10.95$ & $10.95-11.65$ & - \\
\hline
\end{tabular}

profiles for each pixel with the surface air temperature and a scaling factor of the total water vapor amount as two unknown variables by solving a 14-equation set.

Assuming that the atmospheric impacts can be effectively removed, we can therefore focus on estimating both LST and emissivity from multispectral thermal infrared imagery in this study. Both MODIS and ASTER teams have developed new algorithms for separating LST and emissivity from multiple thermal bands [1], [2], which are quite different in nature.

Effects of LST and emissivity on thermal radiance are so closely coupled that their separation from thermal radiance measurements alone is quite difficult. This is because a single multispectral thermal measurement with $N$ bands presents $N$ equations in $N+1$ unknowns ( $N$ spectral emissivities and LST). It is a typical ill-posed inversion problem. Without any prior information, it is impossible for us to recover both LST and emissivity exactly. Most LST-emissivity separation studies used one additional empirical equation so that $N$ measurements plus this additional equation can be solved for $N+1$ unknowns. For example, the Alpha-derived emissivity (ADE) method [18], [19] makes use of the relation between the weighted logarithm values of spectral emissivity and the variance of spectral emissivities. The reference channel method [20] assumes that the value of the emissivity for one of the image channels is constant and known a priori, reducing the number of unknowns to the number of equations.

The comparisons among different LST/emissivity separation algorithms have been well discussed by Gillespie et al. [2], [21] and $\mathrm{Li}$ et al. [22]. Based on previous algorithms, the ASTER team developed a new empirical equation, which was derived by using 86 samples. When more samples were available, the coefficients of this equation varied [2]. Based on nearly 1000 samples from different sources, we found that none of the empirical relationships in the previous studies hold. Fig. 1 shows the relationship between the minimum emissivity and the range of emissivities. There is a tremendous amount of variability in this figure, which may result in a possible failure of such an empirical equation. This demonstrates a need for developing new algorithms. In this study, we developed a new empirical equation. Moreover, our algorithm utilizes an optimal inversion algorithm that has solid foundations in computational mathematics and different regularization techniques for stabilizing the final solutions. In this context, we can easily incorporate any possible prior information and knowledge into this iterative procedure.

\section{NEW APPROACH}

The central idea of the proposed algorithm is very similar to the previous algorithms in the sense that we created a new equa- tion so that $N+1$ equations are used to solve $N+1$ unknown variables. However, since any added equations are based on empirical formulae, the solutions are likely to be unstable. It is a typical ill-posed inversion problem. According to Moritz [23], the inversion problem is called properly posed if the solution satisfies the following requirements: 1 ) the solution must exist (existence); 2) the solution must be uniquely determined by the data (uniqueness); and 3) the solution must depend continuously on the data (stability). If one or more these requirements are violated, then we have an improperly posed, or ill-posed problem. In our present case, it is an ill-posed problem since $N$ observations cannot determine $N+1$ unknowns uniquely. Additional information is needed to turn it to a properly posed problem. One way of providing this additional information is the use of appropriate constraints. This process is known as regularization of the ill-posed problem. Different regularization methods [24], [25] were explored to stabilize the solutions in this study.

Nearly 1000 surface emissivity spectra of different cover types provided by Dr. Salisbury and from the JPL spectral library were integrated to the ASTER and MODIS bands using their sensor spectral response functions. We developed a new empirical relation to predict the minimum emissivity for the MODIS and ASTER, respectively. This inversion algorithm is quite general and suitable for any multispectral thermal infrared remote sensing. We simply take MODIS and ASTER as examples while discussing this new inversion algorithm below, but this method has general applicability.

\section{A. MODIS}

The MODIS sensor has a total of 36 bands and more than a dozen thermal bands. The MODIS science team has proposed to make use of seven bands for LST and emissivity estimation [1]. Based on our MODTRAN simulations, we found that band 33 has very low atmospheric transmittance (lower than 0.35 in many cases) under different atmospheric conditions. We therefore consider only six bands in this study. Their spectral wavelengths are shown in Table I. The spatial resolution of MODIS thermal bands is $1 \mathrm{~km}$, and the scanning angle is as large as $55^{\circ}$. It is carried by Terra (EOS-AM platform) and acquires data at 10:30am local time and will be also carried by the EOS-PM platform acquiring data at 2:30pm local time.

The general empirical equation for the MODIS implementation of our method is given by

$$
\begin{aligned}
\hat{\epsilon}_{\min }= & 0.067+0.319 * \epsilon_{20}+0.232 * \epsilon_{22}+0.271 * \epsilon_{23}+0.381 * \epsilon_{29} \\
& +0.289 * \epsilon_{31}+0.261 * \epsilon_{32}-0.583 * \epsilon_{\text {range }}-0.822 * \epsilon_{\text {med }}
\end{aligned}
$$

where $\epsilon_{\text {med }}$ and $\epsilon_{\text {range }}$ are the median and range of absolute emissivities of 6 bands. The fitted results were very good, with the R-squared value of 0.999 and the residual standard error (RSE) of 0.0073. If the emissivity spectra is quite smooth, the uncertainty associated with (1) does not affect the inversion results significantly. However, it has been realized from the exploratory experiments that the separation of emissivity and LST is highly sensitive to this additional empirical relation. Based on the observations in Fig. 1(a), we established specific empirical 


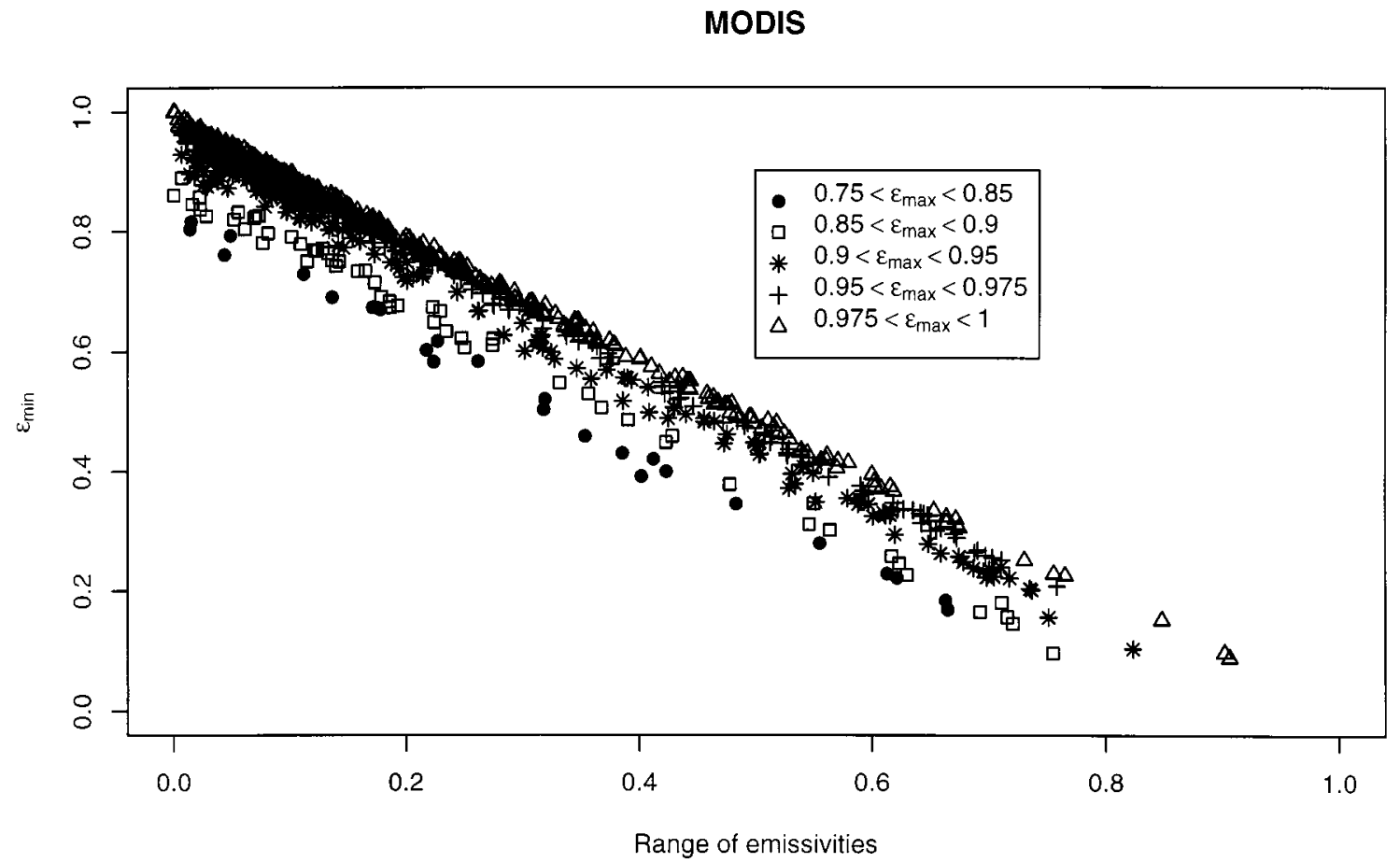

(a)

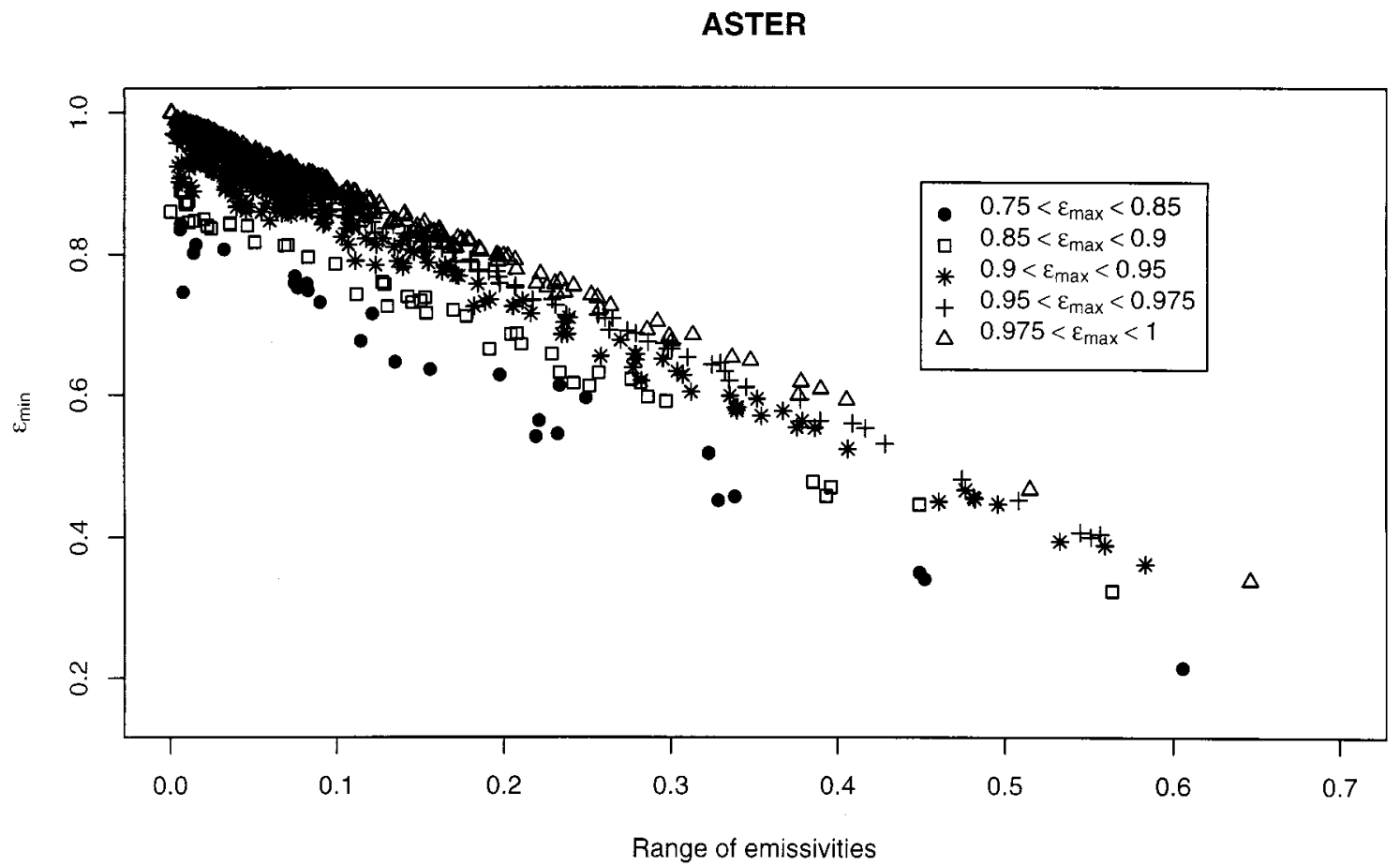

(b)

Fig. 1. Relations between the minimum emissivity and the range of the emissivity values for both (a) MODIS and (b) ASTER. A few outliers in the lower left corner were excluded.

equations for several ranges of the maximum emissivity that had significantly improved the results. If $\epsilon_{\max } \leq 0.85$

$\hat{\epsilon}_{\min }=0.592-0.916 * \epsilon_{\text {range }}+0.083 * \epsilon_{23}+0.129 * \epsilon_{29}+0.082 * \epsilon_{31}$.

If $0.85 \leq \epsilon_{\max }<0.9$

$$
\hat{\epsilon}_{\min }=0.349-0.723 * \epsilon_{\text {range }}-0.643 * \epsilon_{\text {med }}+0.25 * \epsilon_{20}
$$

$$
\begin{aligned}
& +0.133 * \epsilon_{22}+0.225 * \epsilon_{23}+0.263 * \epsilon_{29}+0.16 * \epsilon_{31} \\
& +0.22 * \epsilon_{32} .
\end{aligned}
$$

If $0.9 \leq \epsilon_{\max }<0.95$

$$
\begin{aligned}
\hat{\epsilon}_{\min }= & 0.396-0.741 \epsilon_{\text {range }}-0.595 \epsilon_{\text {med }}+0.185 \epsilon_{20}+0.19 \epsilon_{22} \\
& +0.178 \epsilon_{23}+0.2645 \epsilon_{29}+0.168 \epsilon_{31}+0.185 \epsilon_{32} .
\end{aligned}
$$




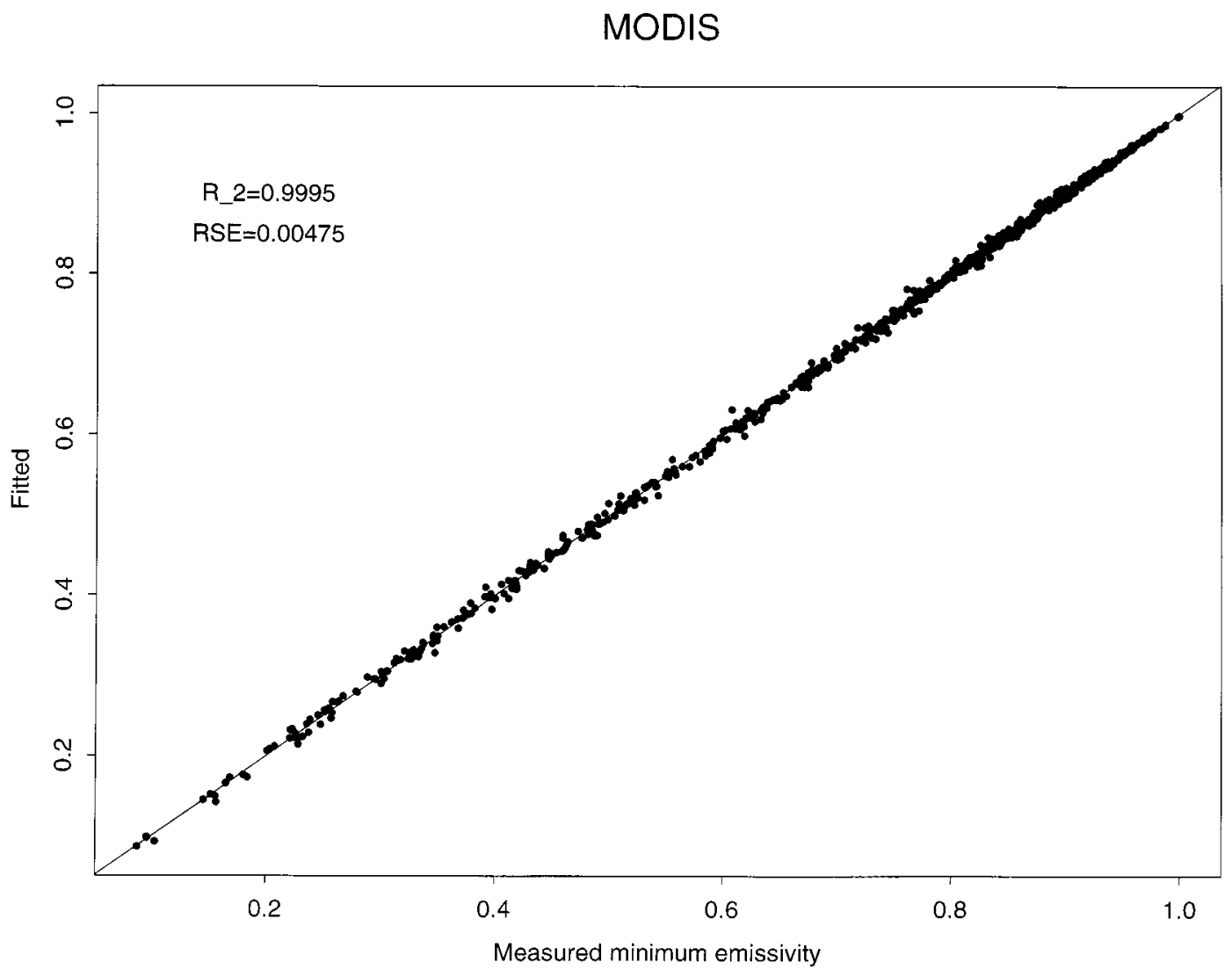

Fig. 2. Fitted minimum emissivity again the actual MODIS minimum emissivity values using (2)-(6).

$$
\begin{aligned}
& \text { If } 0.95 \leq \epsilon_{\max }<0.975 \\
& \begin{aligned}
\hat{\epsilon}_{\min }= & 0.399-0.734 * \epsilon_{\text {range }}-0.473 * \epsilon_{\text {med }}+0.212 * \epsilon_{20} \\
& +0.15 * \epsilon_{22}+0.137 * \epsilon_{23}+0.22 * \epsilon_{29}+0.215 * \epsilon_{31} \\
& +0.127 * \epsilon_{32} .
\end{aligned}
\end{aligned}
$$

$$
\begin{aligned}
& \text { If } 0.975 \leq \epsilon_{\max } \leq 1 \\
& \begin{aligned}
\hat{\epsilon}_{\min }= & 0.175-0.622 * \epsilon_{\text {range }}-0.715 * \epsilon_{\text {med }}+0.246 * \epsilon_{20} \\
& +0.244 * \epsilon_{22}+0.243 * \epsilon_{23}+0.34 * \epsilon_{30}+0.284 * \epsilon_{31} \\
& +0.182 * \epsilon_{32} .
\end{aligned}
\end{aligned}
$$

The fitting results were much better (Fig. 2), and the RSE decreases to 0.0047 .

Any other prior knowledge might help to determine the solutions more accurately. After examining these spectral emissivity spectra, we found many additional relations that can be used to constrain the LST and emissivity separation. These relations can be derived from these constraints, as illustrated in Fig. 3.

\section{B. ASTER}

The ASTER sensor has five thermal bands (see Table I) with the spatial resolution of $90 \mathrm{~m}$, and the scanning angle is less than $8.5^{\circ}$. It is also carried by Terra.

The general empirical equation we developed for the ASTER estimates is given by

$$
\hat{\epsilon}_{\min }=0.0160+0.3098 * \epsilon_{10}+0.2352 * \epsilon_{11}+0.3477 * \epsilon_{12}
$$

$$
\begin{aligned}
& +0.2458 * \epsilon_{13}+0.2862 * \epsilon_{14}-0.5406 * \epsilon_{\text {range }} \\
& -0.4411 * \epsilon_{\text {med }} .
\end{aligned}
$$

The fitting result was similar to the MODIS analysis, with an R-squared value of 0.998 and RSE of 0.0075. Likewise, a further improvement in temperature and emissivity estimates was made by fitting similar equations for several ranges of the maximum emissivity. If $\epsilon_{\max }<0.85$

$$
\begin{aligned}
\hat{\epsilon}_{\min }= & 0.101-0.634 * \epsilon_{\text {range }}-0.326 * \epsilon_{\text {med }}+0.2 * \epsilon_{10} \\
& +0.289 * \epsilon_{11}+0.189 * \epsilon_{12}+0.197 * \epsilon_{13}+0.326 * \epsilon_{14} .
\end{aligned}
$$

If $0.85 \leq \epsilon_{\max }<0.9$

$$
\begin{aligned}
\hat{\epsilon}_{\min }= & 0.44-0.776 * \epsilon_{\text {range }}-0.238 * \epsilon_{\text {med }}+0.164 * \epsilon_{10} \\
& +0.145 * \epsilon_{11}+0.145 * \epsilon_{12}+0.154 * \epsilon_{13}+0.128 * \epsilon_{14} .
\end{aligned}
$$

$$
\begin{aligned}
& \text { If } 0.9 \leq \epsilon_{\max }<0.95 \\
& \qquad \begin{aligned}
\hat{\epsilon}_{\min }= & 0.267-0.664 * \epsilon_{\text {range }}-0.377 * \epsilon_{\text {med }}+0.305 * \epsilon_{10} \\
& +0.117 * \epsilon_{11}+0.287 * \epsilon_{12}+0.171 * \epsilon_{13}+0.211 * \epsilon_{14}
\end{aligned}
\end{aligned}
$$

If $0.95 \leq \epsilon_{\max }<0.975$

$$
\hat{\epsilon}_{\min }=0.441-0.754 * \epsilon_{\text {range }}-0.233 * \epsilon_{\text {med }}+0.169 * \epsilon_{10}
$$$$
+0.151 * \epsilon_{11}+0.164 * \epsilon_{12}+0.123 * \epsilon_{13}+0.17 * \epsilon_{14} \text {. }
$$ 

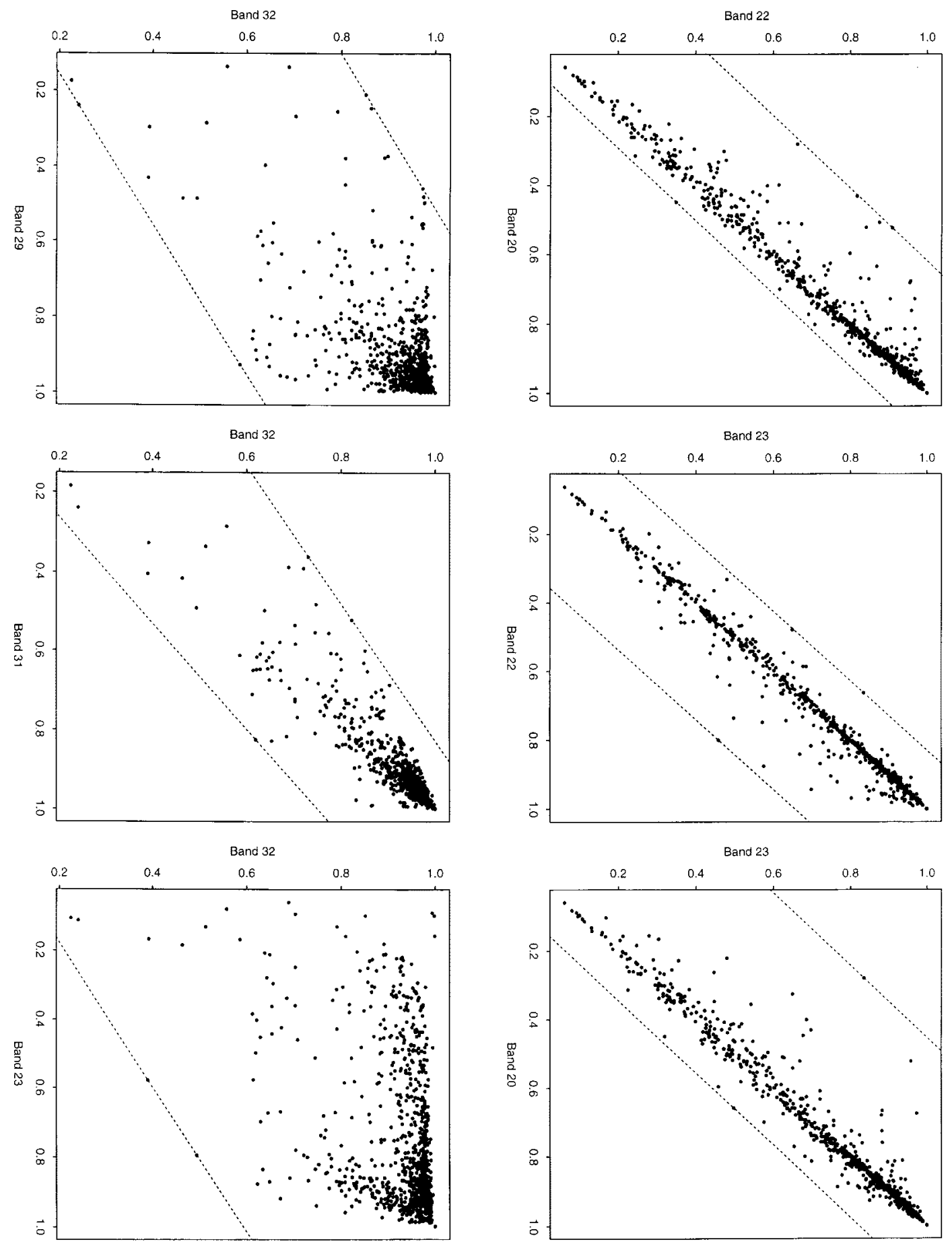

Fig. 3. Illustration of the conditional constraints used for retrieving MODIS surface emissivities. The envelopes banded by two dashed lines indicate the variation ranges of these MODIS band emissivities.

$$
\begin{aligned}
& \text { If } 0.975 \leq \epsilon_{\max } \leq 1 \\
& \begin{aligned}
\hat{\epsilon}_{\min }= & 0.359-0.715 * \epsilon_{\text {range }}-0.312 * \epsilon_{\text {med }}+0.255 * \epsilon_{10} \\
& +0.15 * \epsilon_{11}+0.23 * \epsilon_{12}+0.188 * \epsilon_{13}+0.126 * \epsilon_{14} .
\end{aligned}
\end{aligned}
$$

The results were significantly better (Fig. 4). Similar to the MODIS analysis, we also found many additional constraints based on the range of data values used in the estimations. Some of these constraints are illustrated in Fig. 5.

\section{Inversion Procedure}

To estimate $N+1$ unknowns from $N+1$ nonlinear equations, a multidimensional optimization algorithm is needed. For global applications, computational speed is a major concern. On the other hand, the added empirical equation does not fit the data perfectly. If we treat it as a perfect equation, the uncertainty is introduced into the inversion process. Instead, we developed a constrained one-dimensional (1-D) inversion procedure. The 1-D optimum inversion algorithm for estimating LST is to min- 


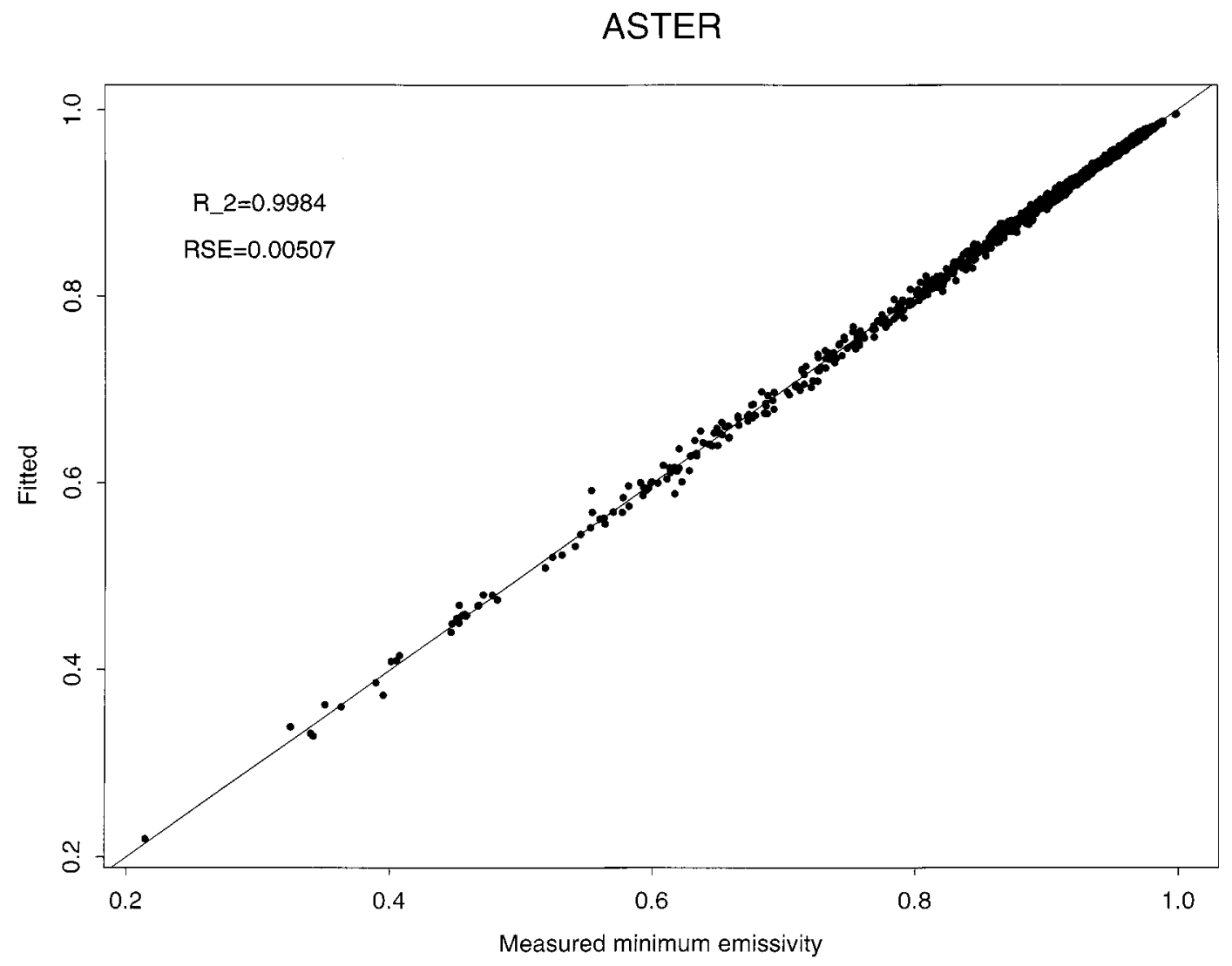

Fig. 4. Fitted minimum emissivity again the actual ASTER minimum emissivity values using (8)-(12).

imize the merit function that consists of a sum of squares (SS) term and a penalty function

$$
F\left(\epsilon_{1,2 \cdots N}, T\right)=S S+\sum f_{i}\left(\epsilon_{1,2 \cdots N}, T\right) 10^{20} .
$$

The SS term is expressed by the smoothness of the retrieved emissivity curve. Different regularization techniques produce different measures of the smoothness [25], [24]. Four regularization techniques were explored in this study

$$
S S= \begin{cases}\sum_{i=1}^{N} \epsilon_{i}{ }^{2} & \text { power } \\ \sum_{i=1}^{N}\left(\epsilon_{i}-\bar{\epsilon}\right)^{2} & \text { variance } \\ \sum_{i=2}^{N}\left(\epsilon_{i-1}-\epsilon_{i}\right)^{2} & \text { first-order difference } \\ \sum_{i=3}^{N}\left(\epsilon_{i-2}-2 \epsilon_{i-1}+\epsilon_{i}\right)^{2} & \text { second-order difference }\end{cases}
$$

where $\epsilon_{i}$ is the emissivity value at band $i, \bar{\epsilon}$ is the mean value of the spectral emissivity curve. This first measure corresponds to the power of the emissivity signal. The second technique is closely related to the first one, which eventually is the variance of the spectral emissivity curve. The third and forth technique is based on the first-order and second-order differences of the emissivity curve.
The penalty function $f_{i}\left(\epsilon_{1,2 \cdots N}, T\right)$ is designed to force all estimated parameters in reasonable ranges. If the value of a variable is beyond its range, a huge penalty is posed (with the huge weight $10^{20}$ ). This huge weight was recommended by Siddall [26]. In our previous studies [27]-[30], we found Siddall algorithm is very effective. In particularly, we can easily incorporate the new empirical equation and other constraints into the penalty term. Rather than requiring a perfect additional equation, we just force all points to be within the envelope around the regression line that defines the empirical equation. For MODIS, if (1) is used the envelope is defined by $-0.03517<\epsilon_{\min }-$ $\hat{\epsilon}_{\min }<0.04455$. If (2)-(6) are used, the envelope is defined by $-0.02488<\epsilon_{\min }-\hat{\epsilon}_{\min }<0.01872$. Similarly, for the ASTER, if (7) is used, for example, $-0.04046<\epsilon_{\min }-\hat{\epsilon}_{\min }<0.02982$. If (8)-(12) are used, $-0.03926<\epsilon_{\min }-\hat{\epsilon}_{\min }<0.02827$. From these envelopes, we can see that the fitting formulae (2)-(6) and (8)-(12) can produce better results.

Estimation of LST and emissivity values from (13) is an typical nonlinear optimization problem that determines the solutions iteratively. The iterative process works as follows. Given an initial LST $\left(T_{0}\right)$, band emissivities are derived based on

$$
\epsilon_{i}=\frac{\left(L_{i}-L_{i}^{s k y}\right)}{L_{i}\left(T_{0}\right)-L_{i}^{s k y}}
$$

where $L_{i}$ and $L_{i}{ }^{s k y}$ are the surface-leaving radiance and downward sky radiance from the atmospheric correction procedure, and $L_{i}\left(T_{0}\right)$ is the blackbody radiance that can be calculated by integrating radiance $B_{i}\left(T_{0}\right)$ using the Planck formula with the 

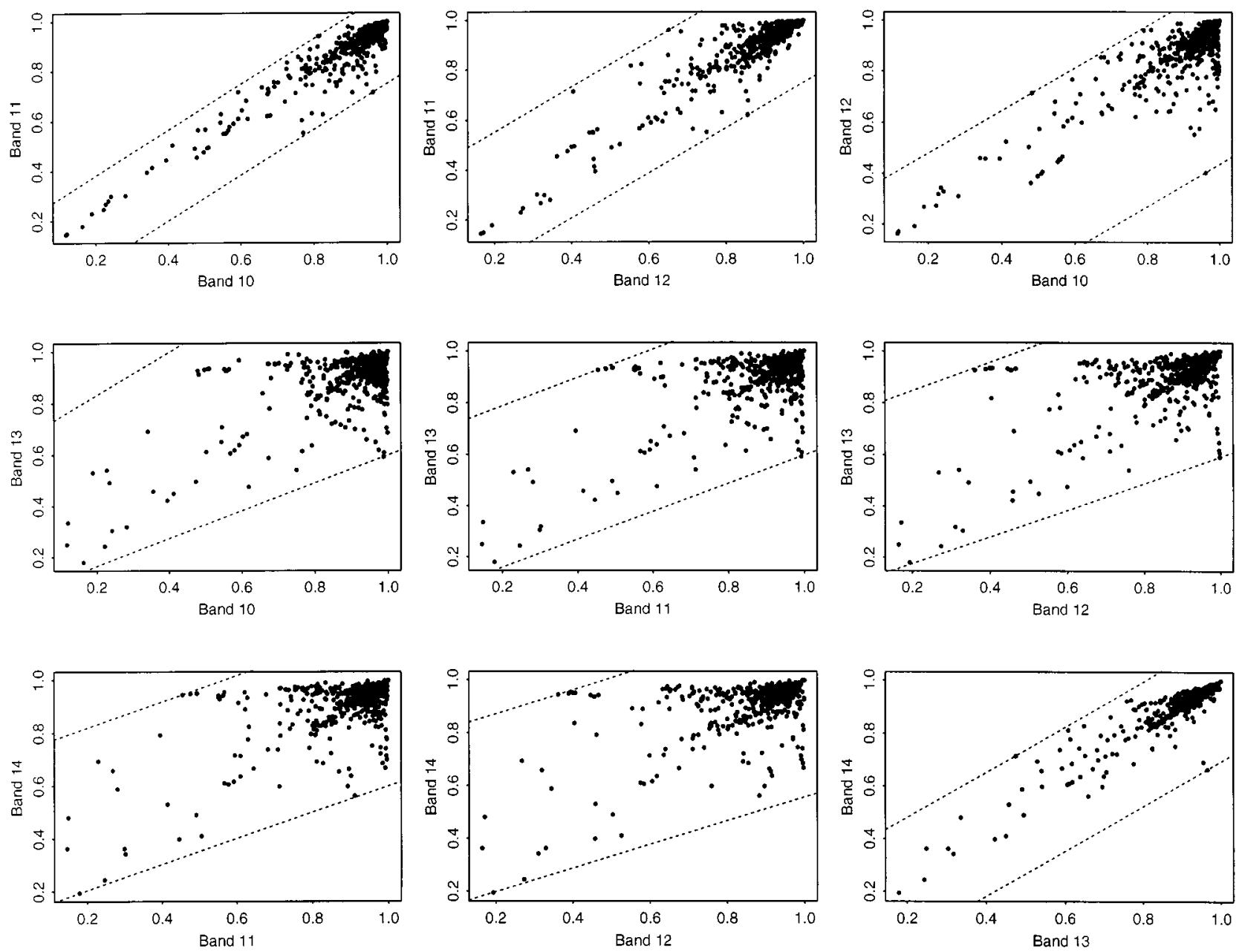

Fig. 5. Illustration of the conditional constraints used for retrieving ASTER surface emissivities. The envelopes banded by two dashed lines indicate the variation ranges of these ASTER band emissivities.

sensor band spectral response functions. The Planck formula for radiance with the unit WATTS $\mathrm{CM}^{-2} \mathrm{STER}^{-1} \mu \mathrm{m}^{-1}$ is given by

$$
B_{i}\left(T_{0}\right)=\frac{1.19095610^{-16} V^{5}}{\exp \left(1.43879 V / T_{0}\right)-1}
$$

where $V$ is the wavenumber $(10000 / \lambda, \lambda$ is the wavelength in $\mu \mathrm{m})$. Note that this algorithm is based on the assumption that observed radiance has been corrected atmospherically. There is therefore no path radiance and transmittance in (15). After determining $\epsilon_{\min }, \epsilon_{\text {med }}$ and $\epsilon_{\text {range }}, \hat{\epsilon}_{\text {min }}$ is predicted based on (1) or (2)-(6) for the MODIS, and (7) or (8)-(12) for the ASTER. The merit function (13) is then calculated. For the next iteration, the iteration length and iteration direction must be found optimally. The iteration continues until the convergence reaches (i.e., $T_{N+1}-T_{N}$ smaller than a threshold).

There are at least four major differences between the present new algorithm and the published algorithms in the literature: 1) the empirical equations are dramatically different; 2) regularization methods are applied; 3) more prior information have been defined and naturally incorporated into the new algorithm in a formal manner; and 4) the new algorithm uses an optimal in- version algorithm that has solid foundations in computational mathematics.

\section{NUMERICAL EXPERIMENTS}

To evaluate the performance of this new approach, we conducted a series of numerical inversion experiments. The numerical experiments serve as the first step for testing any new algorithms. Field experiments ultimately should validate the new approaches, but many conditions (e.g., $\epsilon_{i}$ and $T_{s}$ ) cannot be easily measured in the field. As a result, it is not easy to determine the model accuracy using measured data from the field.

We arbitrarily selected $10 \%$ of the profiles (590 in total) from our atmospheric profile database, which is composed of profiles from the NAVY profile model, and the measured real profiles from the Large Scale Biosphere-Atmosphere Experiment in Amazonia (LBA), Boreal Ecosystems Atmosphere Study (BOREAS), and First ISLSCP Field Experiment (FIFE). LST was then assumed to be equal to the air temperature at the surface level, which varies from $268^{\circ}$ to $307.2^{\circ}$. A total of 937 emissivity spectra of soil, rocks, minerals and vegetation and these atmospheric profiles were input to MODTRAN to simulate downward sky fluxes and surface leaving radiance. A 


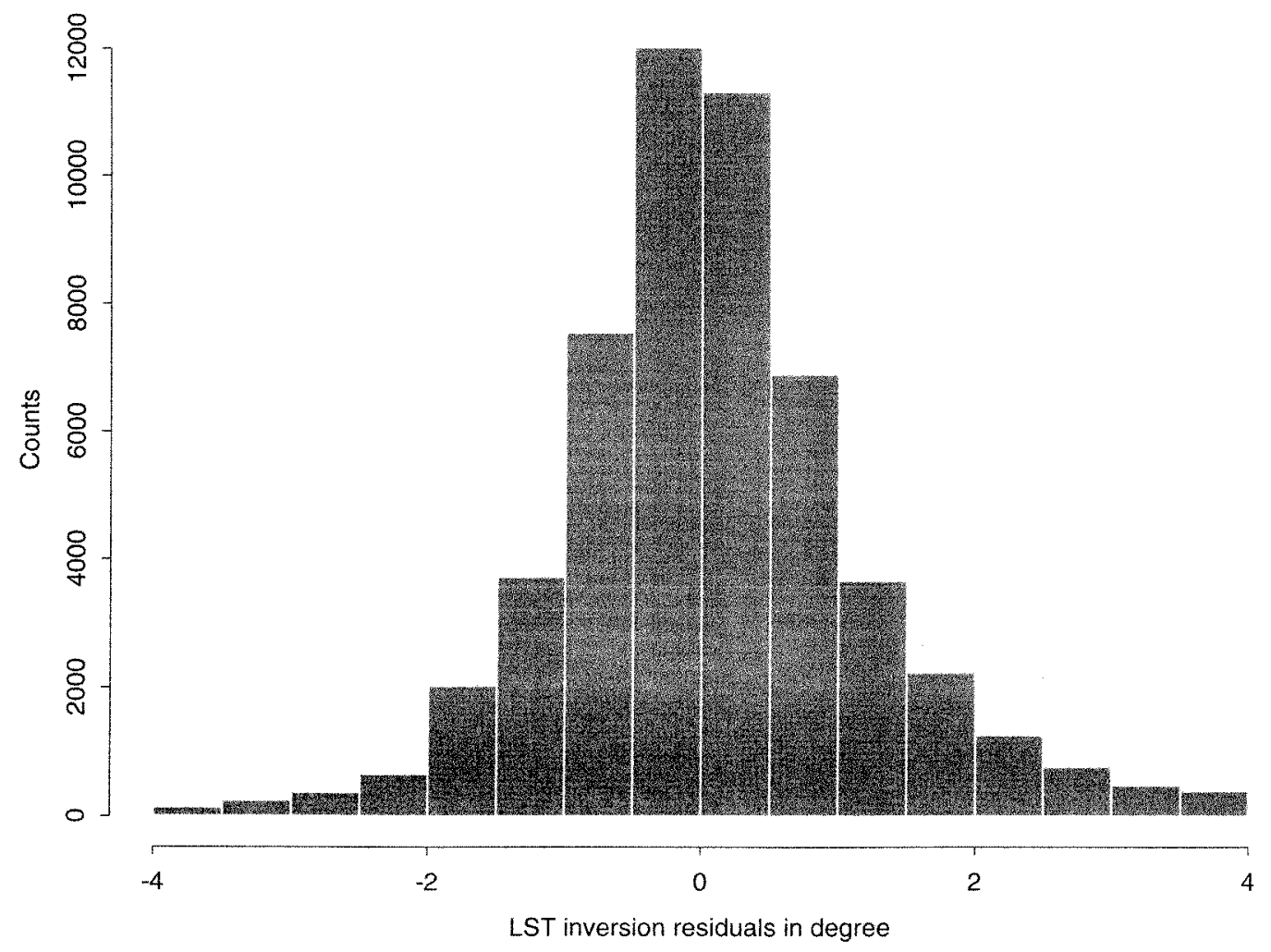

Fig. 6. Histogram of the actual and retrieved LST difference from the simulated MODIS data.

total of 55283 observations for both MODIS and ASTER were generated by integrating the MODTRAN outputs with these sensor spectral response functions.

We compared the performance of these four regularization techniques (14) that were discussed in the previous section. In most cases, the variance measure and the first order difference measure performed the best, and other two techniques performed less well. In the following, all reported results only came from the variance regularization technique using the simulated MODIS data.

The objective of this numerical experiment was to determine if this new inversion algorithm can accurately retrieve the range of surface emissivities and temperature values. The results indicated that we could not inverse both LST and emissivities perfectly. The histogram of the difference between the retrieved LST and actual LST is shown in Fig. 6. However, more than $43.4 \%$ inversion results differed from the actual LST within $0.5^{\circ}, 70.2 \%$ within $1^{\circ}$ and $84 \%$ within $1.5^{\circ}$. The regression between the actual LST $(T)$ and the retrieved LST $(\hat{T})$ was a little biased:

$$
T=6.5651+0.9781 \hat{T}
$$

The R-squared value was 0.98 , and the residual standard error was $1.1^{\circ}$.

The retrieved emissivities of individual bands are displayed in Fig. 7. The residual standard errors ranged from 0.019 at bands 31 and 32 to 0.038 at band 20, the R-squared values varied from
0.912 at band 32 to 0.967 at band 22. Since LST was not perfectly retrieved, the retrieved emissivities also could not match the actual emissivities perfectly.

The errors may come from different sources. The majority of the error is attributed to the empirical equation, which is only a best fit for a given emissivity range. Four cases are presented in Fig. 8 to illustrate this point. The solid lines stand for the actual emissivity curves. In case 1 , two sets of solutions were found, and the retrieved LST was about $15^{\circ}$ from the actual LST. Case 3 was similar, although the LST difference was much smaller. In cases 2 and 4, three sets of solutions were found and they all satisfied the empirical relations.

If we examined these cases carefully, we found that the retrieved emissivity curves look very similar to the actual ones, although their magnitudes might be quite different. More importantly, all emissivity curves increase with wavelength similar to the blackbody emitted radiance, which explains why shifting emissivity curse can still make all $N+1$ equations valid.

It is found that one method can reduce the uncertainties to some extent. One can run the inversion code for $N$ times where $N$ is the number of thermal bands. The median value of the retrieved multiple solution is defined as the final solution. The initial values are determined in this way. Assuming unity emissivity, the initial value is solved from the Planck equation using the central wavelength of that band, which is calculated as $\lambda_{i}=$ $\int_{\lambda_{1}}^{\lambda_{2}} f_{i}(\lambda) \lambda d \lambda / \int_{\lambda_{1}}^{\lambda_{2}} f_{i}(\lambda) d \lambda$ where $f_{i}(\lambda)$ is the sensor spectral response function. Obviously, there are increased computational expenses. 

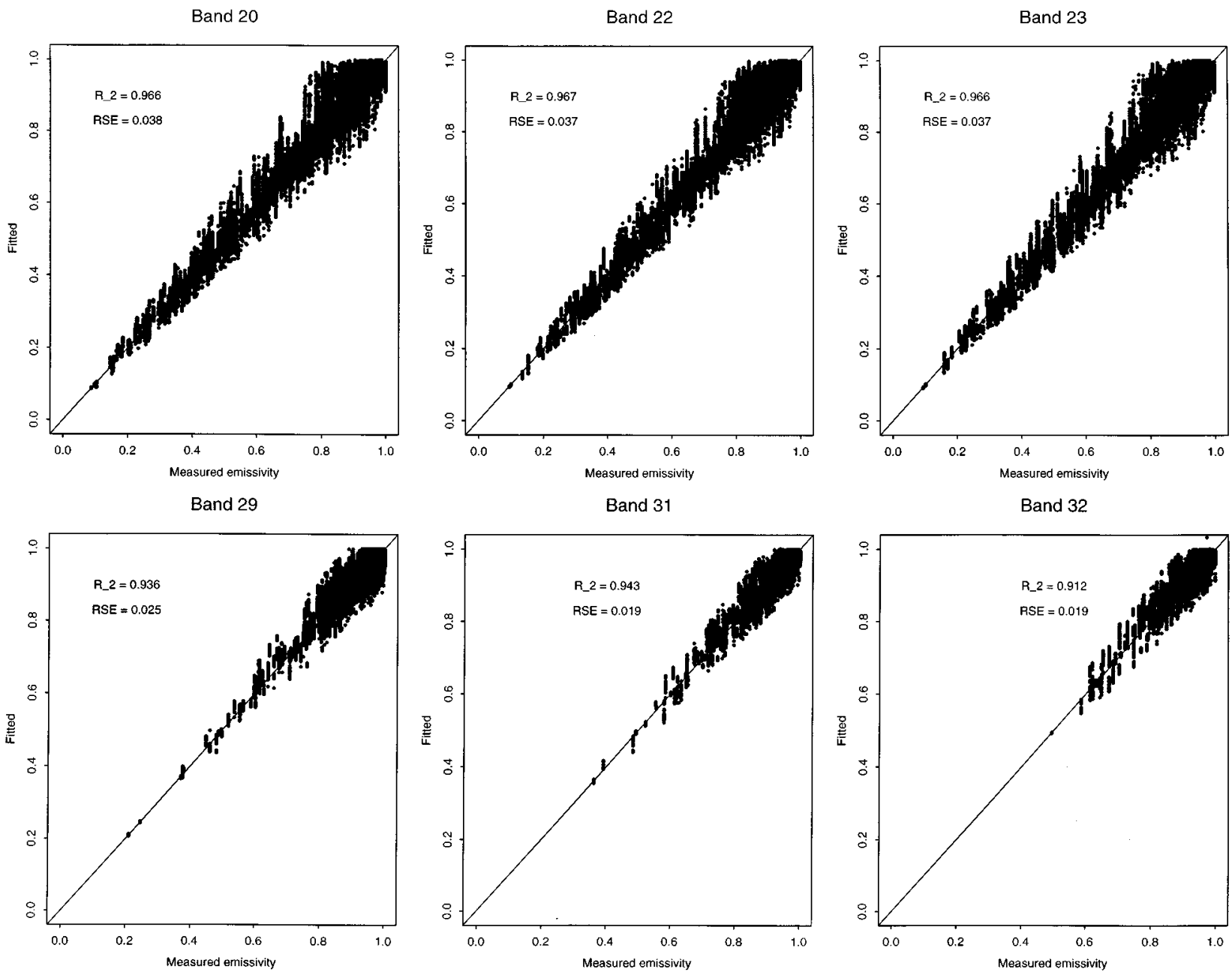

Fig. 7. Retrieved MODIS band emissivities and their actual surface emissivities.

When the same inversion code was run over the simulated ASTER data, the results were very similar. No details are reported here.

\section{SUMMARY}

In this study, we developed a general method for retrieving both LST and emissivity simultaneously from any multispectral thermal infrared imagery. An empirical equation based on the measured emissivity spectra was established. Four different regularization techniques were explored and an optimization algorithm was used to determine the solutions. One of the features of this method is its ability to incorporate any prior knowledge about the spectral emissivities and LST into the inversion algorithm.

Numerical experiments were conducted to test this inversion algorithm. Near 1000 emissivity spectra were integrated into MODIS and ASTER thermal bands in conjunction with the spectral response functions of these sensors. Different atmospheric conditions using a range of LSTs were used to simulate the MODIS and ASTER surface leaving radiance and sky radiance. The optimization code was used to estimate LST and spectral emissivities simultaneously. The results showed that both LST and spectral emissivity can be estimated with a reasonably good accuracy (more than $70.2 \%$ inversion results differed from the actual LST within $1^{\circ}$ ). Two regularization techniques (variance and first-order difference) worked very well, while other two regularization techniques (power and second order difference) worked less well in our experiments.

From these numerical experiments, we found that if the emissivity values of all bands were very low, the solution was very likely to be unstable. In a few cases, the maximum LST difference could be more than $10^{\circ}$. Further algorithm developments are needed to address this issue.

This method works for surface-leaving radiance, an output from the atmospheric correction procedure. The accuracy of atmospheric correction of multispectral thermal infrared imagery will eventually affect the separation of LST and emissivity. The discussion of atmospheric correction is beyond this paper, but it is a critical issue that we need to pay more attention to in the future.

To validate this algorithm using ground measurements, we are also making efforts to apply this algorithm to the airborne multispectral thermal data from the MODIS airborne simulator (MAS) and the airborne thermal infrared multispectral scanner (TIMS) sensors. The results will be reported later. 
Case 1

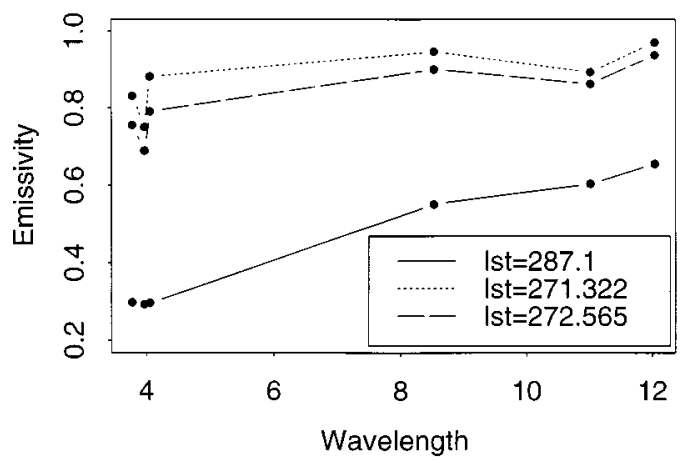

Case 3

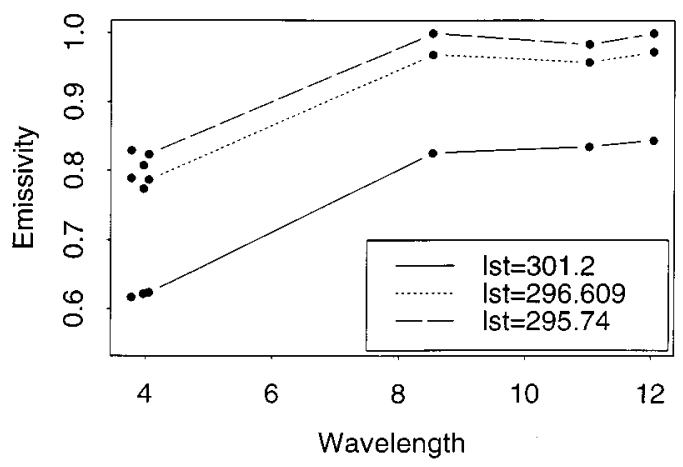

Case 2

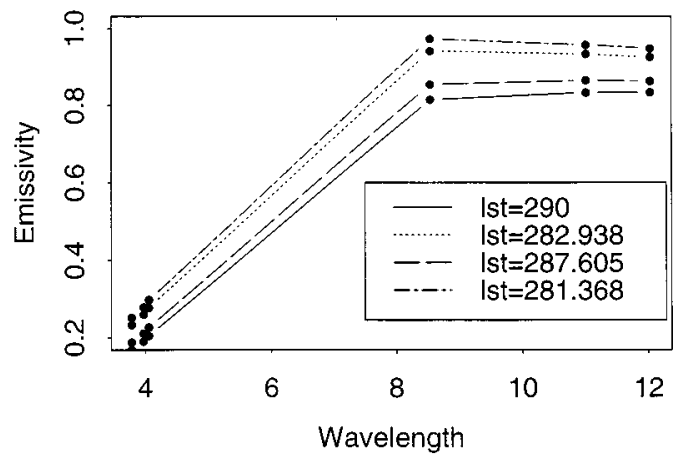

Case 4

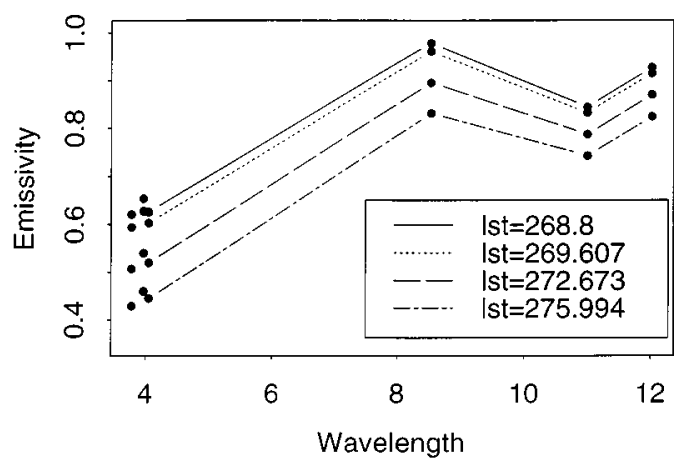

Fig. 8. Illustration of four cases that produce more than seven solutions with six MODIS observations and one additional equation.

\section{ACKNOWLEDGMENT}

The author would like to thank the MODTRAN Development Team, Dr. J. Salisbury for providing the measured emissivity spectra, and the ASTER Science Team for providing the ASTER Spectral Library data. He would also like to thank the anonymous reviewers for their valuable comments, which greatly improved the presentation of this paper.

\section{REFERENCES}

[1] Z. Wan, MODIS Land Surface Temperature Algorithm Theoretical Basis Documentation, Version 3.3, Apr. 1999.

[2] A. R. Gillespie, S. Rokugawa, T. Matsunaga, J. Cothern, S. Hook, and A. Kahle, "A temperature and emissivity separation algorithm for Advanced Spaceborne Thermal Emission and Reflection Radiometer (ASTER) images," IEEE Trans. Geosci. Remote Sensing, vol. 36, pp. 1113-1126, July 1998.

[3] P. Rowntree, "Atmospheric parameterization schemes for evaporation over land: Basic concepts and climate modeling aspects," in Land Surface Evaporation: Measurement and Parameterization, T. Schmugge and J. Abdre, Eds., 1991, pp. 5-34.

[4] J. C. Price, "Land surface temperature measurements from the split window channels of the NOAA-7/AVHRR," J. Geophys. Res., vol. 89, pp. 7231-7237, 1984

[5] Z. M. Wan and J. Dozier, "Land-surface temperature measurement from space: Physical principles and inverse modeling," IEEE Trans. Geosci. Remote Sensing, vol. 27, pp. 268-278, Jan. 1989.

[6] A. J. Prata, "Land surface temperatures derived from the advanced very high resolution ra diometer and the along-track scanning radiometer-1: Theory," J. Geophys. Res., vol. 98, pp. 16689-16702, 1993.

[7] E. P. McClain, W. G. Pichel, and C. C. Walton, "Comparative performance of AVHRR-based multichannel sea surface temperatures," $J$ Geophys. Res., vol. 90, pp. 11 587-11 601, 1985.
[8] A. F. Pearce, A. J. Prata, and C. R. Manning, "Comparison of NOAA/AVHRR-2 sea surface temperatures with surface measurements in coastal waters," Int. J. Remote Sensing, vol. 10, pp. 37-52, 1992.

[9] J. A. Sobrino, C. Coll, and V. Caselles, "Atmospheric corrections for land surface temperature using AVHRR channel 4 and 5," Remote Sens. Environ., vol. 38, pp. 19-34, 1991.

[10] F. Becker and Z. Li, "Surface temperature and emissivity at various scales: Definition, measurement and related problems," Remote Sens. Rev., vol. 12, pp. 225-253, 1995.

[11] A. A. Van De Griend and M. Owe, "On the relationship between thermal emissivity and the normalized difference vegetation index for natural surfaces," Int. J. Remote Sensing, vol. 14, pp. 1119-1131, 1993.

[12] E. Valor and V. Caselles, "Mapping land surface emissivity from NDVI: Application to European, African, and South American areas," Remote Sens. Environ., pt. 1, vol. 57, pp. 67-184, 1996.

[13] W. Snyder, Z. Wan, Y. Zhang, and Y. Feng, "Classification-based emissivity for land surface temperature measurement from space," Int. J. Remote Sensing, vol. 19, pp. 2753-2774, 1998.

[14] A. J. Prata, V. Caselles, C. Coll, J. Sobrino, and C. Ottle, "Thermal remote sensing of land surface temperature from satellites: Current status and future prospects," Remote Sens. Rev., vol. 12, pp. 175-224, 1995.

[15] V. Salomonson, W. Barnes, P. Maymon, H. Montgomery, and H. Ostrow, "MODIS: advanced facility instrument for studies of the Earth as a system," IEEE Trans. Geosci. Remote Sensing, vol. 27, pp. 145-153, Jan. 1989

[16] Y. Yamaguchi, A. Kahle, H. Tsu, T. Kawakami, and M. Pniel, "Overview of advanced spaceborne thermal emission and reflection radiometer (ASTER)," IEEE Trans. Geosci. Remote Sensing, vol. 36, pp. 1062-1071, July 1998.

[17] F. Palluconi, G. Hoover, R. Alley, M. Jentoft-Nilsen, and T. Thompson, An Atmospheric Correction Method for ASTER Thermal Radiometry over Land, Revision 2. Washington, DC: NASA, Aug. 1996.

[18] P. Kealy and S. Hook, "Separating temperature and emissivity in thermal infrared multispectral scanner data: Implication for recovering land surface temperatures," IEEE Trans. Geosci. Remote Sensing, vol. 31, pp. 1155-1164, Nov. 1993. 
[19] S. Hook, A. Gabell, A. Green, and P. Kealy, "A comparison of techniques for extracting emissivity information from thermal infrared data for geological studies," Remote Sens. Environ., vol. 42, pp. 123-135, 1992.

[20] R. J. P. Lyon, "Analysis of rocks by spectral infrared emission (8 to 25 microns)," Econ. Geol., vol. 60, pp. 715-736, 1965.

[21] A. R. Gillespie, S. Rokugawa, S. Hook, T. Matsunaga, and A. Kahle, ASTER Temperature/Emissivity Separation Algorithm Theoretical Basis Document, Version 2.4. Washington, DC: NASA, Mar. 1999.

[22] Z.-L. Li, F. Becker, M. Stoll, and Z. Wan, "Evaluation of six methods for extracting relative emissivity spectra from thermal infrared images," Remote Sens. Environ., vol. 69, pp. 197-214, 1999.

[23] H. Moritz, "General considerations regarding inverse and related problems," in Inverse Problems: Principles and Applications in Geophysics, Technology, and Medicine, G. Anger, R. Gorenflo, H. Jochmann, H. Moritz, and W. Webers, Eds. Berlin, Germany: Akademie Verlag, 1993, pp. 11-23.

[24] S. Twomey, Introduction to the Mathematics of Inversion in Remote Sensing and Indirect Measurements. Mineola, NY: Dover, 1977.

[25] A. Tikhonov and V. Arsenin, Solutions of Ill-Posed Problems. Washington, DC: V. H. Winston and Sons, 1977.

[26] J. N. Siddall, Analytical Decision-making in Engineering Design. Englewood Cliffs, NJ: Prentice-Hall, 1972.

[27] S. Liang and A. H. Strahler, "An analytic canopy radiative transfer BRDF model and its inversion," IEEE Trans. Geosci. Remote Sensing, vol. 31, pp. 1081-1092, Sept. 1993.

[28] S. Liang and J. R. G. Townshend, "Parametric soil BRDF model: A fourstream approximation," Int. J. Remote Sensing, vol. 17, pp. 1303-1315, 1996.
[29] _ - "A modified Hapke model for soil bidirectional reflectance," Remote Sens. Environ., vol. 55, pp. 1-10, 1996.

[30] S. Liang and A. H. Strahler, "Retrieval of surface BRDF parameters from multiangle remotely sensed data," Remote Sens. Environ., vol. 50, pp. $18-30,1994$.

Shunlin Liang (M'94) received the Ph.D. degree in remote sensing and GIS from Boston University, Boston, MA, in 1992

He was a Postdoctoral Research Associate with Boston University from 1992 to 1993, and Validation Scientist of the NOAA/NASA Pathfinder AVHRR Land Project from 1993 to 1994. He is currently an Assistant Professor with the University of Maryland, College Park. His present research interests focus on land surface data assimilation, parameter retrieval from remotely sensed data, and spatial analysis. He is the Principal Investigator of the NASA EOS Terra Validation Team, the NASA Earth Observer-1 Science Team, and the International ALOS, CHRIS/PROBA, and POLDER Science Working Teams. He organized the International Forum on BRDF in San Francisco, CA, in December 1998, and edited a special issue of Remote Sensing Reviews.

Dr. Liang chaired various sessions in the international conferences and serves as chairman of IEEE Geosciences and Remote Sensing Society, Washington/North Virginia Chapter, 2000. 